\title{
VAPOR-PRESSURE RELATIONS IN MIXTURES OF TWO LIQUIDS, III
}

BY A. ERNEST TAYLOR

After obtaining the numerical results of my last paper, it is interesting to see what of theoretical interest they may contain. We expect to find some relation between the composition of the vapor given off and the composition of the solution giving off such vapor. We also expect to find some relation between the partial pressure of acetone in the vapor and the percentage of acetone in the solution or between the partial pressure of the acetone and the pressure of pure acetone at that temperature. The most natural thing to do is to test the Law of Henry and the van 't Hoff-Raoult formula to see if they hold."

The Law of Henry is formulated as follows:

$$
\frac{p_{1}}{c_{1}}=\text { const. }
$$

or

$$
\frac{p_{2}}{c_{2}}=\text { const. }
$$

In Equation I., $p_{1}$ is the partial pressure of the acetone in the vapor, and $c_{\mathrm{r}}$ the corresponding concentration of the acetone in the solution. In Equation II., $p_{2}$ is the partial pressure of the water-vapor, and $c_{2}$ the concentration of the water in the solution. These equations hold at constant temperature. They are both formulations of the same law. In Equation I. acetone is the solvent and in Equation II. water. This simple formation of Henry's law would hold only when in the solution the com-

${ }^{2}$ Jour. Phys. Chem. 4, 355 (1900).

${ }^{2}$ Margules ; Wien. Ber. 104, 1243 (1895); Lehfeldt. Phì. Mag. [5] 46, 46 (1898), 48, 215 (1899). 
pounds under consideration were monomolecular. That acetone exists as simple molecules in aqueons solutions, at least up to concentrations of 30 percent acetone, was shown by the freezingpoint measurements of Waddell. ${ }^{1}$

Equations III. and. IV. represent the familiar van 't HoffRaoult formula

$$
\begin{aligned}
& \log _{\text {nat }} \frac{p_{0}}{p_{1}}=\frac{c_{2}}{c_{1}}=\frac{n}{\mathrm{~N}} \\
& \log _{\text {nat }} \frac{p_{0}}{p_{2}}=\frac{c_{1}}{c_{2}}=\frac{\mathrm{N}}{n}
\end{aligned}
$$

The letters $p_{\mathrm{r}}, p_{2}, c_{\mathrm{r}}, c_{2}$, have the same meaning as before. The vapor-pressure of pure acetone is indicated by $p_{\mathrm{r}}$ and of pure water by $p_{0} . \quad N$ and $n$ represent the relative number of molecules of water and acetone in the solution. By transposing and changing to ordinary logarithms, we get expressions for $p_{2}$ and $p_{\mathrm{r}}$. These relations are more useful in the present consideration, and are given in Equations V. and VI.

$$
\begin{aligned}
& \log _{10} p_{0}-\left(0.4343 \frac{c_{1}}{c_{2}}\right)=\log p_{2} . \\
& \log _{10} p-\left(0.4343 \frac{c_{2}}{c_{1}}\right)=\log p_{1} .
\end{aligned}
$$

As before, the temperature is supposed to be constant, so a set of tables, Table II.-IX. have been made out at constant temperatures from $60^{\circ}-25^{\circ}$ to test Formula V. and Table X. for Formula VI. Table I. gives a set of general constants used throughout the other tables. In these tables the first column gives the gram percentage composition of acetone in the solution, the second the total pressure of the vapor given off, the third the molecular percentage composition of the vapor in acetone, the fourth the same calculated from the formula, the fifth the gram percentage composition of acetone in the solution, the sixth the same, calculated from column four, the seventh the partial pressure of the acetone, the eighth the partial pressure of the water, and the ninth the same calculated from the formula.

${ }^{1}$ Jour. Phys. Chem. 3, I60 (I899). 
In working out the partial pressures of the water and the acetone, it has been assumed that one simple molecular weight of water and one simple molecular weight of acetone both occupy the same volume in the gaseous form; and that in consequence, the partial pressure of each component is proportional to the molecular percentage composition of the vapor, and can be obtained by multiplying this percentage composition by the vaporpressure of the solution. The columns labeled $p_{1}$ and $p_{2}$ were so calculated, that is by multiplying the figures in column two by those in three, the figures in column $p_{\mathrm{I}}$ were obtained; and $p_{s}-p_{\mathrm{r}}=p_{2}$. The figures in $p_{2}$ calc, were obtained from the Formula V. and the percentage and molecular percentage composition of the vapor corresponding determined from them by the following simple formulae:

$$
\begin{aligned}
& \frac{p_{1}}{p_{s}}=\text { acetone percentage mol. concentration of vapor. } \\
& \left(\mathrm{I}-\frac{p_{1}}{p_{s}}\right)=\text { water percentage mol. concentration of vapor. } \\
& \mathrm{I} 8 \frac{p_{1}}{p_{s}}=\mathrm{D} . \quad 5^{8}\left(\mathrm{I}-\frac{p_{1}}{p_{s}}\right)=\mathrm{E} \text {. } \\
& \frac{\mathrm{D}}{\mathrm{D}+\mathrm{E}}=\text { grams of acetone in } \mathrm{I} 00 \mathrm{~g} \text {. of solution. } \\
& \frac{\mathrm{E}}{\mathrm{D}+\mathrm{E}}=\text { grams of water in } \mathrm{I} 00 \mathrm{~g} \text {. of solution. }
\end{aligned}
$$

It will be seen from the tables that the van't Hoff-Raoult equation in general predicts within one or two percent the composition of the vapor given off by any given acetone solution, and this was fully as accurately as I succeeded in determining the composition of the vapor given off. The pressure relations do not agree well, for they depend on the molecular concentration, and an error of one percent on the grani concentration of the vapor introduces an error several times as large in the molecular concentration. This is due to the fact that the molecular weights of water and acetone are so very different, viz., I 8 and 58 . Ninety percent acetone contains only seventysix molecular weights of acetone to one hundred of solution, the 
difference between the two being particularly great at the concentrations most used. An error of two percent in the composition of the vapor would introduce an error of nearly six percent in the vapor composition. When the vapor-pressure is $700 \mathrm{~mm}$ this would introduce an error of $42 \mathrm{~mm}$ in the partial pressure of the water or of the acetone. The partial pressure of the water is not more than $100 \mathrm{~mm}$, so an immense percentage error is introduced. ${ }^{\mathrm{r}}$

It will be seen that there is seldom more than one percent variation between the calculated and the observed vapor decomposition up to sixty percent, after that a little more, and in the ninety percent about three percent difference. This is, I think, well beyond the possible experimental error. It can then well be said that the van 't Hoff-Raoult formula predicts accurately the composition up to sixty percent or perhaps a little higher, and above that there is a tendency for less acetone to be given off than the formula would indicate. This is exactly what one would expect from the formula and where the water acts as solvent. The tables at the lower temperatures do not give quite as good results as the higher ones, as would be expected.

Table X. shows very clearly that Formula VI. does not hold at all, where the acetone is looked upon as solvent.

Tables XI.-XVIII. contain data with reference to Henry's law as expressed in Equations I. and II. The concentrations are all molecular concentrations except in Table XII. The headings of the columns make their meaning sufficiently evident without further explanation. At $55^{\circ}$ in Table XII. the law is tested in a variety of ways. In the fourth column gram concentrations in the solution are used. When the partial pressure of the water is considered $\frac{p_{2}}{c_{2}}$ percent has a constantly rising value; if this is changed to molecular concentrations a reasonably good constant is obtained for the lower percentages, and by taking the square root of the concentrations for the higher concentrations. This was also carried on to the cube root and a constant nearly

1. The apparent increase in the partial pressure of the water on dilution is further evidence of the experimental error. 
as good obtained. On plotting the logarithmic curve, however, the points lie better on the square root curve.

The same methods were employed with $p_{\mathrm{I}}$ the partial pressure of the acetone $\frac{p_{1}}{c_{1}}$ and $\frac{p_{1}}{v_{1}}$ gives very little approach at a constant, but $\frac{p_{1}}{\sqrt[3]{c_{1}}}=$ gives good valves at the lower concentrations and $\frac{p_{1}}{\sqrt[4]{c_{1}}}$ at the higher concentrations. ${ }^{\mathrm{I}}$

The simple formula and the square root formula is used in all the other tables for the water; and the cube root and the fourth root formula for the acetone. The water formula does not hold well for the very highest concentration of acetone, nor the acetone formula for the lowest concentrations, as might be expected.

Not very great accuracy can be expected for this application of Henry's law for the partial pressures are not accurately known, especially those of the water. A more accurate knowledge of the vapor composition is needed before a satisfactory test of the law is made. It is especially unfortunate that the molecular weights of acetone and water are so different, this complicating this part of the work. The fact that the vapor from a rather dilute solution is so rich in acetone makes the accurate determination of the vapor composition almost impossible with any method yet devised. The present data would indicate however that Henry's Law does hold when complex molecules are asassumed for both the water and the acetone in solution.

This paper, then, would seem to indicate that:

The van 't Hoff-Raoult formula, when water is the solvent, predicts, within the limits of experimental error, the composition of the vapor given off from all but the highest concentrations of acetone; and that Henry's law holds only when complex molecular structure is assumed for water and acetone in solution.

${ }^{1}$ Strictly speaking, the values of $c_{1}$ should be recalculated since they are based on the assumption of identity between formula weight and molecular weight; but it seemed hardly worth while to do this with the present data which are in no sense final. 
TABLE I

\begin{tabular}{|c|c|c|c|c|c|c|c|c|}
\hline $\begin{array}{l}\% \\
\text { Ac. }\end{array}$ & $\mathrm{C}_{1}$ & $\log C_{1} \mid$ & $\mathrm{C}_{2}$ & $\log C_{2}$ & $\frac{C_{1}}{C_{2}}$ & $\frac{\mathrm{C}_{2}}{\mathrm{C}_{1}}$ & $\left(0.4343 \frac{C_{1}}{C_{2}}\right)$ & $\left(0.4343 \frac{\mathrm{C}_{2}}{\mathrm{C}_{1}}\right)$ \\
\hline O & o. & & 100 & 2.0000 & - & - & - & \\
\hline IO & $3 \cdot 3$ & $0.5^{185}$ & 96.7 & I. 9854. & $0.034 \mathrm{I}$ & 29.3 & 0.0148 & I 2.7 \\
\hline 20 & 7.2 & 0.8573 & 92.8 & I. 9675 & 0.0775 & I 2.9 & 0.0337 & 5.598 \\
\hline 30 & II. 7 & 1.0582 & 88.3 & 1.9460 & $0.13^{2}$ & 7.55 & 0.0575 & 3.278 \\
\hline 40 & $\mathrm{I} 7 . \mathrm{I}$ & $\left|\mathrm{I} .233^{\circ}\right|$ & 82.9 & 1.9186 & 0.206 & 4.85 & 0.0896 & 2.106 \\
\hline 50 & 23.7 & I. 3747 & 76.3 & 1.8825 & 0.310 & 3.22 & O. I349 & I. 398 \\
\hline 60 & 31.8 & 1.5024 & 68.2 & I. 8338 & 0.466 & 2.14 & 0.2025 & $0.93=6$ \\
\hline 70 & 42.0 & 1.6232 & 58.0 & I. 7634 & 0.724 & 1.38 & 0.3145 & 0.5958 \\
\hline 80 & 55.4 & I.7435 & 44.6 & I. 6493 & 1.24 & 0.805 & 0.5395 & 0.3496 \\
\hline 90 & 73.6 & I. 8669 & 26.4 & 1.4216 & 2.79 & 0.359 & $\mathrm{I} .2 \mathrm{II}$ & 0.1558 \\
\hline 100 & 100 & $|2.0000|$ & 0.0 & & & & & \\
\hline
\end{tabular}

TABLE II

$60^{\circ}$

$\log { }_{149}=2.173^{2}$

\begin{tabular}{|c|c|c|c|c|c|c|c|c|}
\hline $\begin{array}{l}\% \\
\text { Ac. }\end{array}$ & $\begin{array}{c}p_{s} \\
\mathrm{~mm}\end{array}$ & $\begin{array}{l}\text { Mols. Ac } \\
\text { vapor }\end{array}$ & $\begin{array}{l}\text { Ibic. } \\
\text { calc. }\end{array}$ & $\begin{array}{l}\% \text { Ac. } \\
\text { vapor }\end{array}$ & $\begin{array}{l}\text { Ibid. } \\
\text { calc. }\end{array}$ & $\underset{\text { mim. }}{p_{1}}$ & $\underset{\mathrm{m} m}{p_{2}}$ & $\begin{array}{c}p_{2} \text { calc. } \\
\text { mm. }\end{array}$ \\
\hline o & I 49 & o & 0 & o & 0 & 0 & 149 & 149 \\
\hline IO & 339 & 56.0 & $57 \cdot 5$ & 80.8 & 8I 5 & 190 & 149 & 144 \\
\hline 20 & 485 & 70.5 & $71: 5$ & 88.5 & 89 & 342 & 143 & 138 \\
\hline 30 & 577 & 76.8 & $77 \cdot 5$ & $9 \mathrm{I} .5$ & 9 I. 8 & 443 & 134 & I $3 \mathrm{I}$ \\
\hline 40 & 640 & $77 \cdot 3$ & $8 \mathrm{I}$ & 9 I. 8. & $93 \cdot 3$ & 495 & I 45 & I 2 I \\
\hline 50 & 682 & 81.2 & 84 & 93.3 & 94.5 & 553 & I 29 & 109 \\
\hline 60 & 714 & 82.3 & 87 & 93.8 & 95.5 & 588 & I 26 & 93.5 \\
\hline 70 & 740 & 84.3 & 90.3 & 94.5 & 96.8 & 624 & I I 6 & 72 \\
\hline 80 & 774 & 86.8 & 94.5 & 95.5 & 98 & 672 & 102 & 43 \\
\hline 90 & 808 & 88.0 & 99 & 96 & 99.5 & $7 \mathrm{II}$ & 97 & 9.2 \\
\hline 100 & 860 & 100 & 100 & 100 & 100 & $860^{\circ}$ & 0 & 0 \\
\hline
\end{tabular}


Vapor-pressure Relations in Mixtures of Two Liquids 68I

\section{TABLE III}

$$
55^{\circ}
$$

$\log I_{17.5}=2.0700$

\begin{tabular}{|c|c|c|c|c|c|c|c|c|}
\hline $\begin{array}{l}\% \\
\mathrm{Ac} .\end{array}$ & $\underset{\mathrm{mm}}{p_{s}}$ & $\begin{array}{c}\text { Mols Ac. } \\
\text { vapor }\end{array}$ & $\begin{array}{l}\text { Ibid. } \\
\text { calc. }\end{array}$ & $\begin{array}{l}\% \text { Ac. } \\
\text { vapor }\end{array}$ & $\begin{array}{l}\text { Ibid. } \\
\text { calc. }\end{array}$ & $\begin{array}{c}p_{1} \\
\mathrm{~mm} .\end{array}$ & $\begin{array}{c}p_{2} \\
\mathrm{~m} \mathrm{~m}\end{array}$ & $\begin{array}{c}p_{2} \text { calc. } \\
\mathrm{mm}\end{array}$ \\
\hline 0 & I I 7.5 & 0 & 0 & o & 0 & 0 & I I 7.5 & I I 7.5 \\
\hline IO & 27.5 & 57 & $5^{8.7}$ & $8 \mathrm{I}$ & 82.2 & I 57 & I 8 & I $13 \cdot 5$ \\
\hline 20 & 399 & 7 I. 5 & 72.8 & 89.0 & 89.5 & 283.5 & I I 5.5 & 109 \\
\hline 30 & 478 & $77 \cdot 3$ & 78.5 & 91.8 & 92.3 & 370.5 & 107.5 & 103 \\
\hline 40 & 534 & 78.7 & 82 & 92.3 & 93.8 & 420 & I I & 95.5 \\
\hline 50 & 572 & 82.5 & 85 & 93.8 & 94.8 & 472 & 100 & 86 \\
\hline 60 & 598 & 83 & 87.7 & 94 & 95.8 & 496.5 & IOI. 5 & 73.7 \\
\hline 70 & 621 & 85 & 90.8 & 94.8 & 97 & 528 & 93 & 57.0 \\
\hline 80 & 545 & 87.5 & 94.8 & 95.8 & 98.3 & 566.5 & 78.5 & 34.0 \\
\hline 90 & 680 & 89 & 99 & 96.3 & 99.5 & 602 & 78 & 7.2 \\
\hline 100 & $72 \mathrm{I}$ & 100 & 100 & 100 & IOO & $72 \mathrm{I}$ & 0 & o \\
\hline
\end{tabular}

\section{TABLE IV}

$$
50^{\circ}
$$

\begin{tabular}{|c|c|c|c|c|c|c|c|c|}
\hline $\begin{array}{l}\% \\
\text { Ac. }\end{array}$ & $\underset{\mathrm{nim}}{p_{s}}$ & $\begin{array}{c}\text { Mols Ac. } \\
\text { vapor }\end{array}$ & $\begin{array}{l}\text { Ibid. } \\
\text { calc. }\end{array}$ & $\begin{array}{l}\% \text { Ac. } \\
\text { vapor }\end{array}$ & $\begin{array}{l}\text { Ibid. } \\
\text { calc. }\end{array}$ & $\cdot \underset{\mathrm{mm}}{p_{1}}$ & $\underset{\mathrm{mim}}{p_{2}}$ & $\begin{array}{c}p_{2} \text { calc. } \\
\text { mm. }\end{array}$ \\
\hline 0 & 92 & 0 & o & o & o & 0 & 92 & 92. \\
\hline IO & $22 \mathrm{I}$ & 57 & 59.8 & 81 & 82.8 & I 26 & 95 & 89 \\
\hline 20 & 324 & 71.8 & 73.7 & 89.3 & 90 & 232.5 & $9 \mathrm{~F} .5$ & 85 \\
\hline 30 & 393 & 78 & 79.5 & 92 & 92.5 & 306.5 & 86.5 & 80.5 \\
\hline 40 & 442 & 79.3 & 83 & 92.5 & 94 & 350.5 & 91.5 & 75 \\
\hline 50 & 472 & 83 & 85.7 & 94 & 95 & 392 & 80 & $67 \cdot 5$ \\
\hline 60 & 499 & 83 & 88.5 & 94 & 96 & $4 I_{4}$ & 85 & 58 \\
\hline 70 & 517.5 & 85.5 & 91.3 & 95 & 97 & 442.5 & 75 & $44 \cdot 5$ \\
\hline 80 & 536 & 89 & 95 & 96.3 & 98.5 & 477 & 59 & 26.5 \\
\hline 90 & 566.5 & 89.8 & 99 & 96.5 & 99.8 & 509 & $57 \cdot 5$ & $5 \cdot 7$ \\
\hline 100 & 607 & 100 & 100 & 100 & IOO & 607 & 0 & o \\
\hline
\end{tabular}

$\log 92=\mathrm{I} .9638$ 
TABLE V

$$
45^{\circ} \text {. }
$$

$\log 71.5=\mathrm{I} .8543$

\begin{tabular}{|c|c|c|c|c|c|c|c|c|}
\hline Ac. & $\underset{\mathrm{mm}}{p_{s}}$ & $\begin{array}{c}\text { Mols. Ac } \\
\text { vapor }\end{array}$ & $\begin{array}{l}\text { Ibid. } \\
\text { calc. }\end{array}$ & $\begin{array}{l}\% \text { Ac. } \\
\text { vapor }\end{array}$ & $\begin{array}{l}\text { Ibid. } \\
\text { calc. }\end{array}$ & $\underset{\mathrm{mm}}{p_{1}}$ & $\underset{\mathrm{mm}}{p_{2}}$ & $\begin{array}{l}p_{2} \text { calc. } \\
\text { mm. }\end{array}$ \\
\hline 0 & $7 \mathrm{I} .5$ & 0 & 0 & o & 0 & 0 & 71.5 & 7 I. 5 \\
\hline IO & I 77 & 57 & $6 I$ & $8 \mathrm{I}$ & 83.5 & IOI & 76 & 69 \\
\hline 20 & 262 & 72.5 & 74.8 & 89.5 & 90.5 & I92.5 & 69.5 & 66 \\
\hline 30 & 319 & $79 \cdot 3$ & 81.3 & 92.5 & 93.3 & 253 & 66 & 62.5 \\
\hline 40 & 364 & 80.5 & 84 & 93 & 94.3 & 293 & $7 \mathrm{I}$ & 58 \\
\hline 50 & $39 \mathrm{I}$ & 84.3 & 86.5 & 94.5 & 95.5 & 329.5 & 61.5 & 52.5 \\
\hline 60 & $4 \mathrm{I} 4$ & 83.7 & 89.2 & 94.3 & 96.3 & 346.5 & $67 \cdot 5$ & 45 \\
\hline 70 & 428 & 86.3 & 92 & 95.5 & 97.3 & $369^{\circ}$ & 59 & 34.5 \\
\hline 80 & 447 & 89.5 & 95.5 & 96.5 & 98.5 & 400 & 47 & 20.5 \\
\hline 90 & 469 & 90.3 & 99 & 96.8 & 99.7 & 423.5 & $45 \cdot 5$ & $4 \cdot 4$ \\
\hline 100 & 505 & 100 & 100 & 100 & 100 & 505 & 0 & 0 \\
\hline
\end{tabular}

\section{TABLE VI}

$40^{\circ}$

$\log 55=\mathrm{I} .7404$

\begin{tabular}{|c|c|c|c|c|c|c|c|c|}
\hline $\begin{array}{l}\% \\
\text { Ac. }\end{array}$ & $\underset{\mathrm{m}}{p_{s}}$ & $\begin{array}{c}\text { Mols. Ac } \\
\text { vapor }\end{array}$ & $\begin{array}{l}\text { Ibid. } \\
\text { calc. }\end{array}$ & $\begin{array}{l}\% \text { Ac. } \\
\text { vapor }\end{array}$ & $\begin{array}{l}\text { Ibid. } \\
\text { calc. }\end{array}$ & $\underset{\mathrm{mm}}{p_{1}}$ & $\underset{\mathrm{mm}}{p_{2}}$ & $\begin{array}{c}p_{2} \text { calc. } \\
\mathrm{mm} .\end{array}$ \\
\hline 0 & 55 & 0 & 0 & 0 & 0 & 0 & 55 & 55 \\
\hline IO & I 39 & $57 \cdot 5$ & 61.8 & $8 \mathrm{I} \cdot 3$ & $8_{4}$ & 80 & 59 & 53 \\
\hline 20 & 209 & 73 & 76.7 & 89.8 & 91.5 & I 52.5 & 56.5 & $5 \mathrm{I}$ \\
\hline 30 & $25^{\circ}$ & 79.8 & 81.3 & 92.8 & 93.3 & 206 & $5^{2}$ & 48 \\
\hline 40 & 298.5 & 81.2 & 84.7 & 93.3 & 94.7 & 242.5 & 55.5 & 46 \\
\hline 50 & 319 & 85 & 87.3 & 94.8 & 95.7 & $27 \mathrm{I}$ & 48 & 40.5 \\
\hline 60 & 340 & 84.3 & 84.8 & 94.5 & 96.5 & 286.5 & 53.5 & 34.5 \\
\hline 70 & 352 & 86.8 & 92.5 & 95.5 & 97.5 & 305 & 47 & 26.5 \\
\hline 80 & 368 & 90.3 & 95.7 & 96.8 & 98.7 & $33^{2.5}$ & 35.5 & I 6 \\
\hline 90 & 387.5 & 90.3 & 99.2 & 96.8 & 99.7 & $35^{\circ}$ & 37.5 & 3.5 \\
\hline 100 & 416 & 100 & 100 & IOO & 100 & 416 & 0 & 0 \\
\hline
\end{tabular}


Vapor-pressure Relations in Mixtures of Two Liquids 683

\section{TAble VII}

$$
35^{\circ}
$$

$\log 42=1.623^{2}$

\begin{tabular}{|c|c|c|c|c|c|c|c|c|}
\hline $\begin{array}{l}\% \\
\text { Ac. }\end{array}$ & $\underset{\mathrm{mm}}{p_{s}}$ & $\begin{array}{l}\text { Mols. Ac } \\
\text { vapor }\end{array}$ & $\begin{array}{l}\text { Ibid. } \\
\text { calc. }\end{array}$ & $\begin{array}{l}\% \text { Ac. } \\
\text { vapor }\end{array}$ & $\begin{array}{l}\text { Ibid. } \\
\text { calc. }\end{array}$ & $\underset{\mathrm{mm}}{p_{1}}$ & $\underset{\text { min. }}{p_{2}}$ & $\begin{array}{c}p_{2} \text { calc. } \\
\mathrm{mm} .\end{array}$ \\
\hline o & 42 & o & 0 & 0 & 0 & 0 & 42 & 42 \\
\hline IO & 107 & 57.8 & 62 & 81.5 & 84 & 62 & 45 & 40.5 \\
\hline 20 & I 65 & 73.8 & 76.5 & 90 & 91.3 & I 22 & 43 & 39 \\
\hline 30 & 206 & 80.5 & 82.5 & 93 & 93.7 & I 66 & 40 & 37 \\
\hline 40 & 239 & 82.3 & 85.7 & 93.8 & 95 & 196.5 & 42.5 & 34 \\
\hline 50 & 260 & 86.3 & 88.2 & $95 \cdot 3$ & 96 & 224.5 & 35.5 & $3 \mathrm{I}$ \\
\hline 60 & 277 & 84.3 & 90.5 & 94.5 & 96.8 & 233.5 & 43.5 & 26.5 \\
\hline 70 & 290 & 87.5 & 93.0 & 95.8 & $97 \cdot 7$ & 254 & 36 & 20.5 \\
\hline 80 & 301 & 9 I. 7 & 96 & 97.3 & 98.8 & 276 & 25 & I 2 \\
\hline 90 & 318 & 91.7 & 99.2 & 97.3 & 99.8 & 292 & 26 & 2.5 \\
\hline 100 & 343 & IOO & IOO & IOO & IOO & 343 & o & 0 \\
\hline
\end{tabular}

\section{TABLE VIII}

$30^{\circ}$

$\log 3 \mathrm{I} .5=\mathrm{I} .4983$

\begin{tabular}{|c|c|c|c|c|c|c|c|c|}
\hline $\begin{array}{r}\% \\
\mathrm{AC}\end{array}$ & $\underset{\mathrm{mm}}{p_{s}}$ & $\begin{array}{l}\text { Mols. Ac } \\
\text { vapor }\end{array}$ & $\begin{array}{l}\text { Ibid. } \\
\text { calc. }\end{array}$ & $\begin{array}{l}\% \mathrm{Ac} . \\
\text { vapor }\end{array}$ & $\begin{array}{l}\text { Ibid. } \\
\text { calc. }\end{array}$ & $\underset{\mathrm{mm}}{p_{1}}$ & $\begin{array}{c}p_{2} \\
\mathrm{~mm}\end{array}$ & $\begin{array}{c}p_{2} \text { calc. } \\
\text { mim. }\end{array}$ \\
\hline 0 & $3 I .5$ & 0 & o & 0 & o & o & $3^{\mathrm{I}} \cdot 5$ & $3 \mathrm{I} \cdot 5$ \\
\hline IO & 82 & 57.8 & 62.8 & $8 \mathrm{I} .5$ & 84.5 & 47.5 & 34.5 & 30.5 \\
\hline 20 & 130 & 73.8 & 77.5 & 90 & 91.8 & 96 & 34 & 29 \\
\hline 30 & I 64.5 & $8 \mathrm{I} \cdot 3$ & 83.3 & 93.3 & 94 & I 34 & 30.5 & $27 \cdot 5$ \\
\hline 40 & I 9 I. 5 & 82.3 & 86.5 & 93.8 & 95.5 & I 57.5 & 34 & $25 \cdot 5$ \\
\hline 50 & $2 I I$ & 86.3 & 89 & $95 \cdot 3$ & 96.3 & I 82 & 29 & 23 \\
\hline 60 & 224 & $84 \cdot 3$ & 91.3 & 94.5 & 97 & 189 & 35 & 20. \\
\hline $7^{\circ}$ & 235 & 87.5 & 93.5 & 95.8 & 97.8 & 205 & 29.5 & I 5 . \\
\hline 80. & 245 & 91.7 & 96.3 & 97.3 & 98.7 & 225 & 20 & 9 \\
\hline 90 & 258 & $9 \mathrm{I} .7$ & 99.3 & $97 \cdot 3$ & 99.8 & 236.5 & $2 \mathrm{I} .5$ & 2 \\
\hline 100 & $28 \mathrm{I}$ & 100 & 100 & 100 & 100 & $28 \mathrm{I}$ & 0 & 0 \\
\hline
\end{tabular}


TABLe IX

$25^{\circ}$

$\log 23.5=1.37$ II

\begin{tabular}{|c|c|c|c|c|c|c|c|c|}
\hline 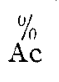 & $\underset{\mathrm{m} m}{p}$ & $\begin{array}{c}\text { Mols, Ac } \\
\text { vapor }\end{array}$ & $\begin{array}{l}\text { Ibid. } \\
\text { calc. }\end{array}$ & $\begin{array}{l}\% \text { Ac. } \\
\text { vapor }\end{array}$ & $\begin{array}{l}\text { Ibid. } \\
\text { calc. }\end{array}$ & $\underset{\mathrm{mm}}{p_{1}}$ & $\begin{array}{c}p_{2} \\
\text { mum. }\end{array}$ & $\begin{array}{c}p_{2} \text { calc. } \\
\text { tumb. }\end{array}$ \\
\hline o & 23.5 & 0 & 0 & O & 0 & 0 & 23.5 & 23.5 \\
\hline IO & $6_{5}$ & $5^{8}$. & 65. & 81.8 & 85.8 & $3^{8}$ & 27 & 22.7 \\
\hline 20 & IO4 & 74 & 79 & 90.3 & 92.5 & 77 & 27 & 21.7 \\
\hline 30 & I 30 & $8 \mathrm{I} \cdot 3$ & 84.3 & 93.3 & 94.5 & 105 & .25 & 20.5 \\
\hline 40 & I 5 I & 83 & $87 \cdot 3$ & 94 & 95.8 & I 25.5 & 24.5 & I9 \\
\hline 50 & I 68 & 86.8 & 89.8 & $95: 5$ & 96.5 & 146 & 22 & I 7 \\
\hline 60 & 180 & 84.2 & $9 \mathrm{I} .8$ & 94.5 & $97 \cdot 3$ & I 52 & 28 & I 5 \\
\hline 70 & I 88 & 87.5 & 94 & 95.8 & 98 & 164.5 & $23 \cdot 5$ & I I 5 . \\
\hline 80 & I98 & 91.8 & 96.5 & $97 \cdot 3$ & 98.8 & I 82 & I 6 & 6.8 \\
\hline 90 & 209 & 9I.8 & $99 \cdot 3$ & $97 \cdot 3$ & 99.8 & 192 & I7 & I. 5 \\
\hline 100 & 229 & IOO & IOO & IOO & 100 & 229 & 0 & 0 \\
\hline
\end{tabular}

TABLe $\mathrm{X}$

$55^{\circ}$

\begin{tabular}{|c|c|c|c|c|c|}
\hline$\%$ Ac. & $p_{s}$ & $\begin{array}{l}\text { Mols. Ac. } \\
\text { vapor }\end{array}$ & Ibid, calc. & $\begin{array}{l}\% \text { Ac. } \\
\text { vapor }\end{array}$ & $\begin{array}{l}\text { Ibid. } \\
\text { calc. }\end{array}$ \\
\hline o & I I 7.5 & o & & o & - \\
\hline IO & 275 & 57 & & $8 \mathrm{I}$ & \\
\hline 20 & 399 & 71.5 & $0 . \mathrm{O}_{5} 455$ & 89 & \\
\hline 30 & 478 & $77 \cdot 3$ & $0.0_{3} 79$ & 91.8 & \\
\hline 40 & 534 & 78.7 & 1.0 & 92.3 & \\
\hline 50 & 572 & 82.5 & 5.0 & 93.8 & 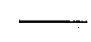 \\
\hline 60 & 598 & 83 & I 4.0 & 94 & 34.5 \\
\hline 70 & 621 & 85 & 29.2 & 94.8 & $57 \cdot 2$ \\
\hline 80 & 645 & 87.5 & 50 & 95.8 & 76.3 \\
\hline 90 & 680 & 89 & 74 & 96.3 & 90.3 \\
\hline 100 & 721 & IOO & 100 & 100 & IOO \\
\hline
\end{tabular}


Vapor-pressure Relations in Mixtures of Two Liquids 685

\section{TABLE XI}

$60^{\circ}$

\begin{tabular}{|c|c|c|c|c|c|c|c|c|}
\hline $\begin{array}{c}\% \\
\text { Ac. }\end{array}$ & $c_{1}$ & $c_{22}$ & $\underset{\text { มnm. }}{p_{1}}$ & $\underset{\mathrm{mm}}{p_{2}}$ & $\frac{p_{1}}{\sqrt[3]{c_{1}}}$ & $\frac{p_{1}}{\sqrt[4]{c_{1}}}$ & $\frac{p_{2}}{c_{2}}$ & $\frac{p_{2}}{\sqrt{c_{2}}}$ \\
\hline 0 & o & 100 & o & I 49 & - & - & I.49 & 14.9 \\
\hline IO & $3 \cdot 3$ & 96.7 & I9O & 149 & 103.5 & I 4 I & I. 54 & I 5.2 \\
\hline 20 & 7.2 & 92.8 & 342 & I 43 & I 77 & 209 & I. 54 & 14.8 \\
\hline 30 & I I.7 & 88.3 & 443 & I 34 & I 95 & 240 & 1.52 & $\mathrm{r} 4.3$ \\
\hline 40 & I $7 . I$ & 82.9 & 495 & I 45 & 192 & 243 & I. 77 & I 5.9 \\
\hline $5^{\circ}$ & 23.7 & 76.3 & 553 & I 29 & I92 & $25^{\circ}$ & 1.69 & I 4.8 \\
\hline 60 & 31.8 & 68.2 & 588 & I 26 & 185 & 247 & I. 85 & I 5.3 \\
\hline 70 & 42.0 & 58.0 & 624 & I I 6 & I79 & 245 & 2.00 & I 5.2 \\
\hline 80 & 55.4 & 44.6 & 672 & 102 & I 76 & 246 & 2.29 & I 5.3 \\
\hline 90 & 73.6 & 26.4 & $7 \mathrm{I} \mathrm{I}$ & 97 & 170 & 243 & 3.67 & I 8.9 \\
\hline IOO & 100 & 0 & 860 & 0 & 185 & 272 & - & - \\
\hline
\end{tabular}




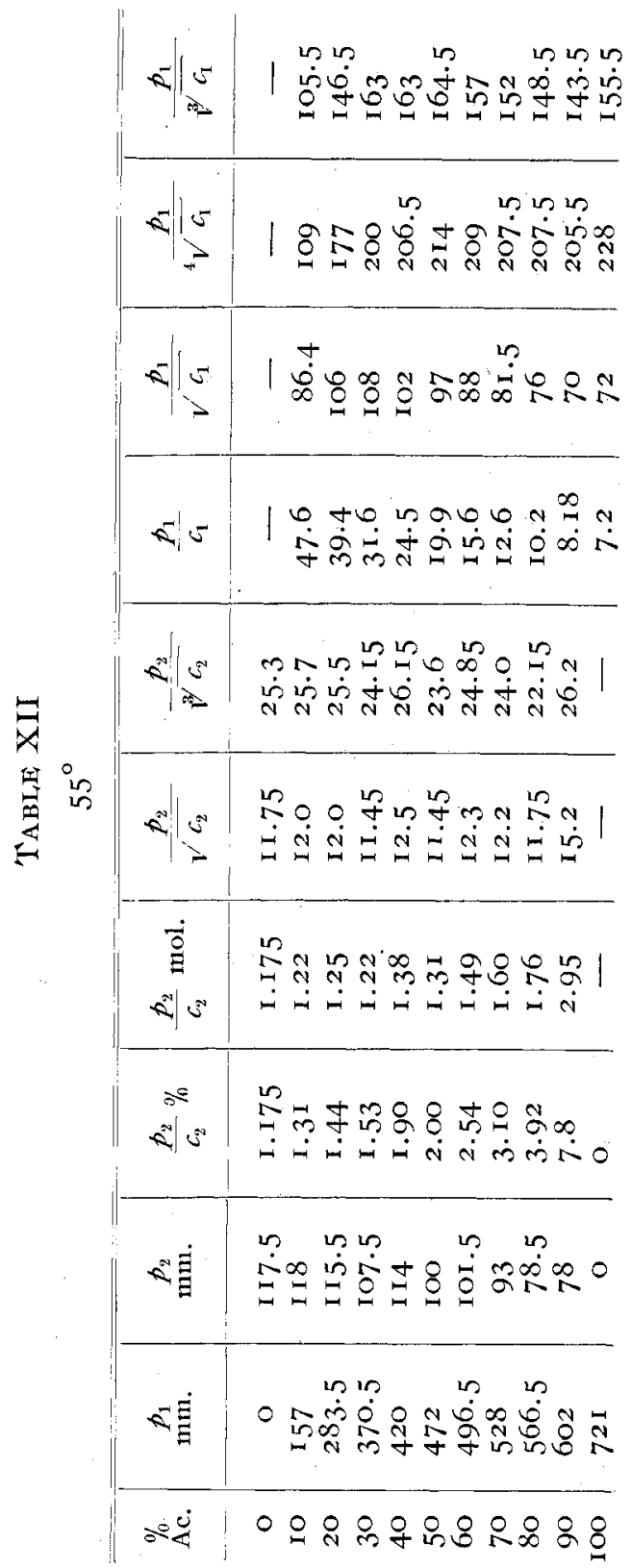


Vapor-pressure Relations in Mixtures of Two Liquids 687

\section{TABLE XIII}

$50^{\circ}$

\begin{tabular}{|c|c|c|c|c|c|c|c|c|}
\hline $\begin{array}{l}\% \\
\text { Ac. }\end{array}$ & $c_{1}$ & $c_{2}$ & $\begin{array}{c}p_{1} \\
\text { mm }\end{array}$ & $\begin{array}{c}p_{2} \\
\mathrm{~mm}\end{array}$ & $\frac{p_{1}}{\sqrt[3]{c_{1}}}$ & $\frac{p_{1}}{\sqrt[4]{c_{1}}}$ & $\frac{p_{2}}{c_{2}}$ & $\frac{p_{2}}{\sqrt{c_{2}}}$ \\
\hline 0 & 0 & 100 & o & 92 & - & - & 0.920 & 9.20 \\
\hline IO & $3 \cdot 3$ & 96.7 & I 26 & 95 & 84.6 & 93.5 & 0.982 & 9.66 \\
\hline 20 & 7.2 & 92.8 & $23^{2} .5$ & $9 \mathrm{I} .5$ & I 20 & I 42 & 0.986 & 9.50 \\
\hline 30 & II .7 & 88.3 & 306.5 & 86.5 & I 35 & I66 & 0.975 & 9.20 \\
\hline 40 & I7. I & 82.9 & 350.5 & $9 \mathrm{II} 5$ & 136 & I 72 & I. IO & 10.05 \\
\hline 50 & 23.7 & 76.3 & 392 & 80 & 136 & I 78 & I.05 & 9.16 \\
\hline 60 & $3^{1.8}$ & 68.2 & $4 \mathrm{I} 4$ & 85 & I $3 \mathrm{I}$ & 174 & I.25 & 10.3 \\
\hline 70 & 42.0 & 58.0 & $44^{2} .5$ & 75 & I 27 & 174 & I.29 & 9.85 \\
\hline 80 & $55 \cdot 4$ & 44.6 & 477 & 59 & 125 & I 75 & 1.32 & 8.84 \\
\hline 90 & 73.6 & 26.4 & 509 & 57.5 & $\mathrm{I} 2 \mathrm{I}$ & I 74 & 2.18 & I I. 2 \\
\hline 100 & 100 & O & 607 & 0 & $\mathrm{I} 3 \mathrm{I}$ & 192 & - & \\
\hline
\end{tabular}

TABLE XIV

\begin{tabular}{|c|c|c|c|c|c|c|c|c|}
\hline $\begin{array}{l}\% \\
\text { Ac. }\end{array}$ & $c_{1}$ & $c_{2}$ & 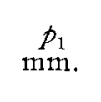 & $\underset{\mathrm{m}}{p_{2}}$ & $\frac{p_{1}}{\sqrt[3]{c_{1}}}$ & $\frac{p_{1}}{\sqrt[4]{c_{1}}}$ & $\frac{p_{2}}{c_{2}}$ & $\frac{p_{2}}{\sqrt{c_{2}}}$ \\
\hline o & o & 100 & 0 & 71.5 & - & - & 0.715 & 7.15 \\
\hline IO & $3 \cdot 3$ & 96.7 & IOI & & 67.7 & 74.7 & 0.786 & 7.15 \\
\hline 20 & 7.2 & 92.8 & I92.5 & 69.5 & 99.7 & I 8 & 0.749 & 7.73 \\
\hline 30 & II. 7 & 88.3 & 253 & 66 & III. 5 & I 37 & 0.747 & 7.22 \\
\hline 40 & 17.1 & 82.9 & 293 & $7 \mathrm{I}$ & II 4 & I 44 & 0.856 & 7.02 \\
\hline $5^{\circ}$ & 23.7 & 76.3 & 329.5 & $6 \mathrm{I}$. & I I 5 & I 49 & 0.806 & 7.80 \\
\hline 60 & $3 \mathrm{I} .8$ & 68.2 & 346.5 & 67.5 & IOS & I.t6 & 0.990 & 7.04 \\
\hline 70 & 42.0 & 58.0 & 369 & 59 & 106 & I 45 & I.04 & 8.17 \\
\hline 80 & 55.4 & 44.6 & 400 & 47 & 105 & I 47 & 1.05 & 7.75 \\
\hline 90 & 73.6 & 26.4 & 423.5 & 45.5 & IOI & I 45 & I. 72 & 7.04 \\
\hline IOO & 100 & 0 & 505 & 0 & IO9 & 160 & & 8.86 \\
\hline
\end{tabular}


TABLE XV

$40^{\circ}$

\begin{tabular}{|c|c|c|c|c|c|c|c|c|}
\hline $\begin{array}{c}\% \\
\text { Ac. }\end{array}$ & $c_{1}$ & $c_{2}$ & $\underset{\mathrm{mm}}{p_{1}}$ & $\underset{\mathrm{mm}}{p_{2}}$ & $\frac{p_{1}}{\sqrt[3]{c_{1}}}$ & $\frac{p_{1}}{\sqrt[4]{V} c_{1}}$ & $\frac{p_{2}}{c_{2}}$ & $\frac{p_{2}}{\sqrt{c_{2}}}$ \\
\hline 0 & 0 & 100 & 0 & 55 & - & - & $0.55^{\circ}$ & $5.5^{\circ}$ \\
\hline IO & $3 \cdot 3$ & 96.7 & 80 & 59 & 53.7 & 59.3 & 0. & 6.00 \\
\hline 20 & 7.2 & 92.8 & I 52.5 & $5^{6.5}$ & 79.0 & 93.1 & 0.609 & 5.87 \\
\hline 30 & I I. 7 & 88 & 206 & 52 & 90.7 & I I I & 0.589 & 5.53 \\
\hline 40 & I $7 . \mathrm{I}$ & 82.9 & 242.5 & 55.5 & $94 . I$ & I I 9 & 0.669 & 6.I6 \\
\hline 50 & 23.7 & 76.3 & $27 \mathrm{I}$ & 48 & 94.4 & I 23 & 0.629 & 5.50 \\
\hline 60 & 31.8 & 68 & 286.5 & 53. & 90.5 & I 2 I & 0.784 & 6.48 \\
\hline 70 & 42.0 & 58.0 & 305 & 47 & 87.9 & 120 & 0.810 & 6.17 \\
\hline 80 & 55.4 & 44.6 & 332.5 & 35.5 & 87.2 & I 22 & 0.795 & $5 \cdot 32$ \\
\hline 90 & 73.6 & 26.4 & 350 & 37.5 & 83.5 & I 20 & 1.42 & $7 \cdot 30$ \\
\hline 100 & IOO & 0 & 416 & 0 & 89.6 & 131 & & - \\
\hline
\end{tabular}

TABLE XVI

$35^{\circ}$

\begin{tabular}{|c|c|c|c|c|c|c|c|c|}
\hline $\begin{array}{l}\% \\
\text { Ac. }\end{array}$ & $c_{1}$ & $c_{2}$ & $\underset{\mathrm{mm}}{p_{1}}$ & $\begin{array}{c}p_{2} \\
\text { min. }\end{array}$ & $\frac{p_{1}}{\sqrt[3]{c_{1}}}$ & $\frac{p_{1}}{\sqrt[4]{c_{1}}}$ & $\frac{p_{2}}{c_{2}}$ & $-\frac{p_{2}}{\sqrt{c_{2}}}$ \\
\hline 0 & o & 100 & o & 4 & 一 & - & 0.42 & 4.2 \\
\hline IO & $3 \cdot 3$ & 96.7 & 62 & 4 & $4^{I} \cdot 5$ & 45.8 & 0.465 & $4 \cdot 5^{8}$ \\
\hline 20 & 7.2 & 92.8 & I 22 & 43 & 63.1 & $74 \cdot 3$ & 0.463 & $4 \cdot 4^{6}$ \\
\hline 30 & I I.7 & 88.3 & I 66 & 40 & 73.0 & 89.7 & 0.453 & 4.26 \\
\hline 40 & I 7.1 & 82.9 & I96.5 & 42. & 76.3 & 96.8 & 0.513 & 4.67 \\
\hline $5^{\circ}$ & 23.7 & 76.3 & 224.5 & 35 & 78.1 & 102 & 0.465 & 4.06 \\
\hline 60 & 31.8 & 68.2 & 233. & 43 & 73.7 & 98.3 & 0.638 & 5.27 \\
\hline 70 & 42.0 & 58.0 & 254 & 36 & 73.0 & 99.6 & 0.620 & 4.73 \\
\hline 80 & 55.4 & 44.6 & 276 & 25 & 72.4 & IOI & 0.560 & 3.74 \\
\hline 90 & 73.6 & 26.4 & 292 & 26 & 69.6 & 99.6 & 0.985 & 5.06 \\
\hline 100 & 100 & o & 343 & O & 73.9 & 108 & - & - \\
\hline
\end{tabular}


Vapor-pressure Relations in Mixtures of Two Liquids 689

TABLE XVII

$30^{\circ}$

\begin{tabular}{|c|c|c|c|c|c|c|c|c|}
\hline $\begin{array}{l}\% \\
\text { Ac. }\end{array}$ & $c_{1}$ & $c_{2}$ & $\begin{array}{c}p_{1} \\
\text { mm. }\end{array}$ & $\begin{array}{c}p_{2} \\
\text { min. }\end{array}$ & $\frac{p_{1}}{\sqrt[3]{c_{1}}}$ & $-\frac{p_{1}}{c_{1}}$ & $\frac{p_{2}}{c_{2}}$ & $\frac{p_{2}}{\sqrt{c_{2}}}$ \\
\hline 0 & o & 100 & o & 31.5 & - & - & 0.315 & 3.15 \\
\hline IO & $3 \cdot 3$ & 96.7 & $47 \cdot 5$ & 34.5 & 31.8 & 35.2 & 0.357 & $3.5 \mathrm{I}$ \\
\hline 20 & 7.2 & 92.8 & 96 & 34 & 49.7 & 58.6 & 0.366 & 3.53 \\
\hline 30 & I I. 7 & 88.3 & I 34 & 30.5 & 58.9 & 72.3 & 0.345 & 3.25 \\
\hline 40 & $17 . \mathrm{I}$ & 82.9 & I 57.5 & 34 & 61.2 & 77.5 & 0.410 & 3.73 \\
\hline $5^{\circ}$ & 23.7 & 76.3 & I82 & 29 & 63.4 & 82.5 & 0.380 & $3.3^{2}$ \\
\hline 60 & 31.8 & 68. & I 89 & 35 & 70.7 & 943 & 0.513 & 4.24 \\
\hline 70 & 42.0 & 58.0 & 205 & 29.5 & 59.2 & 80.8 & 0.509 & 3.87 \\
\hline 80 & 55.4 & 44.6 & 225 & 20 & 58.9 & 82.4 & 0.448 & 2.99 \\
\hline 90 & 73.6 & 26.4 . & $23^{6} .5$ & 21.5 & 56.5 & 80.8 & 0.814 & 4. I8 \\
\hline IOO & IOO & o & $28 \mathrm{I}$ & 0 & 60.6 & 88.9 & 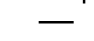 & 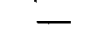 \\
\hline
\end{tabular}

TABLE XVIII

$25^{\circ}$

\begin{tabular}{|c|c|c|c|c|c|c|c|c|}
\hline $\begin{array}{l}\% \\
\text { Ac. }\end{array}$ & $c_{1}$ & $c_{2}$ & $\underset{\mathrm{mm}}{p_{1}}$ & $\begin{array}{c}p_{2} \\
\text { mm. }\end{array}$ & $\frac{p_{1}}{i^{3 / c_{1}}}$ & $\frac{p_{1}}{\sqrt[4]{\sqrt{c_{1}}}}$ & $\frac{p_{2}}{c_{2}}$ & $\frac{p_{3}}{\sqrt{c_{2}}}$ \\
\hline o & 0 & 100 & o & 23.5 & -- & $\cdots$ & 0.235 & 2.35 \\
\hline IO & $3 \cdot 3$ & 96.7 & 38 & 27 & 25.3 & 28.0 & 0.279 & 2.75 \\
\hline 20 & 7.2 & 92.8 & 77 & 27 & 39.8 & 47.0 & $0.29 \mathrm{I}$ & 2.80 \\
\hline 30 & II 7 & 88.3 & 105 & 25 & 46.4 & 57.0 & 0.283 & 2.66 \\
\hline 40 & $17 . \mathrm{I}$ & 82.9 & 125.5 & 24.5 & 48.7 & $6 \mathrm{I} .6$ & 0.296 & 2.69 \\
\hline 50 & 23.7 & 76.3 & 146 & 22 & 50.8 & 66. I & 0.288 & $2.5^{2}$ \\
\hline 60 & $3^{1.8}$ & 68.2 & I $5^{2}$ & 28 & 47.9 & 63.9 & 0.4 I I & 3.39 \\
\hline 70 & 42.0 & 58.0 & I 64.5 & 23.5 & $47 \cdot 3$ & 64.6 & 0.405 & 3.09 \\
\hline 80 & 55.4 & 44.6 & 182 & 16 & 47.7 & 66.6 & 0.370 & 2.47 \\
\hline 90 & 73.6 & 26.4 & 192 & I 7 & 45.8 & 65.5 & 0.663 & 3.33 \\
\hline 100 & 100 & 0 & 229 & 0 & 49.3 & 72.4 & & \\
\hline
\end{tabular}

Cornell University 